\title{
Qualitätsvolles Lehrhandeln im Französischunterricht \\ Mehrsprachigkeitsdidaktik und Kompetenzentwicklung auf der elementaren Bildungsstufe
}

\section{Bettina Imgrund}

Wie können qualitätsvolles Lehrhandeln und die Entwicklung von Kompetenzen im Französischunterricht klarer beschrieben werden? Zur Beantwortung dieser Frage werden zunächst theoretische Modelle und ausgewählte empirische Erkenntnisse aus der Unterrichtsforschung und der Fremdsprachendidaktik vorgestellt. Aus einer Stichprobe von acht Fällen werden danach zwei Fälle, in denen Lehrpersonen mit Prinzipien der Mehrsprachigkeitsdidaktik arbeiten, deskriptiv-interpretativ analysiert. Lernende ziehen darin zum gleichen Lerngegenstand einen jeweils unterschiedlichen Nutzen. Aus einer Gegenüberstellung der Fälle und verschiedener Datenquellen können empirisch fundierte Aussagen zu einem kompetenzunterstützenden Lehrhandeln im elementaren Französischunterricht abgeleitet werden.

\section{Einleitung}

Kompetenzorientierung heisst das Zauberwort, das auch der oft beklagten Situation des Französischunterrichts neuen Schwung verleihen soll. Der Begriff Kompetenz hat dabei stets eine positive Konnotation. Kompetenzen sind realitätsnahe, aufgabenbezogene Handlungsfähigkeiten, welche Lernende als Nutzen aus Unterricht ziehen sollen. Dieser Nutzen umfasst idealerweise fachliche, volitionale und soziale Aspekte (Weinert, 2001). Für den Fremdsprachenunterricht sind die zu erwerbenden Kompetenzen im Europäischen Referenzrahmen für Sprachen klar ausgewiesen (Europarat, 2001), wobei empirische Belege dazu, wie sich Kompetenzen entwickeln und welche unterrichtsbezogenen Qualitätsmerkmale zum gewünschten schülerseitigen Nutzen führen, erst spärlich sind (Schneuwly, 2009).

Nachfolgend soll deshalb der Frage nachgegangen werden, wie ein kompetenzunterstützendes Lehrhandeln für den elementaren Französischunterricht besser beschrieben werden kann und welche unterrichtlichen Zusammenhänge die Kompetenzentwicklung fördern. Lernpsychologisch setzt das Konzept der Kompetenzunterstützung bei Lehrpersonen Diagnosekompetenzen und Kompe- 
tenzen zur Lernunterstützung voraus (Klieme, Pauli \& Reusser, 2005). Beide äussern sich im Unterrichtsgeschehen darin, dass schülerseitige Vorkenntnisse aktiv in den Lehr-Lern-Prozess einbezogen werden und neues Wissen passgenau für die zu unterrichtende Klasse aufgebaut wird. Ähnliche und gleichlautende Kriterien finden sich in der Mehrsprachigkeitsdidaktik, welche vorsieht, die Sprachkenntnisse von Lernenden in den Sprachlehr- und -lernprozess miteinzubeziehen, um so das Interesse für Fremdsprachen zu wecken (Imgrund, 2007; Meissner \& Reinfried, 1998).

Der Artikel präsentiert zwei Fallstudien aus einer Studie mit insgesamt acht Primarschulklassen (Imgrund, 2015) und legt seinen Schwerpunkt auf empirische Evidenz. Die beiden Kontrastfälle eignen sich besonders dazu, Aspekte der Mehrsprachigkeitsdidaktik wie auch der fachlichen, volitionalen und sozialen Kompetenzentwicklung aufzuzeigen sowie bedeutsame tiefenstrukturelle Prozessmerkmale aufzudecken, die in einem Zusammenhang mit der Qualität der Unterrichtsgestaltung stehen. In den Fallstudien wird das französische Alphabet eingeführt und in die Kompetenz «Buchstabieren» überführt. Analysiert wird die Interaktion zwischen Lehrperson und Klasse im Aufbauund im Festigungszyklus, d.h. in einem kurzen Längsschnitt. Zur Analyse von Unterrichtstranskripten werden Auszüge aus einem Interview mit Lernenden zu deren Kompetenzerleben erklärend hinzugezogen. Aus der Analyse des beobachteten und erlebten schülerseitigen Nutzens wird schliesslich auf die Qualität des Unterrichtsangebots geschlossen. Ziel des Artikels ist, Qualitätsmerkmale der lehrseitigen Kompetenzunterstützung für den elementaren Französischunterricht klarer zu konturieren und darüber hinaus einen Beitrag zur Weiterentwicklung der Forschungsmethodologie in der fachdidaktischen Unterrichtsforschung zu leisten. ${ }^{1}$

\section{Theoretisch-empirische Prämissen und Arbeitsfragen}

Neuere Unterrichtsforschung wird häufig auf der Grundlage von sozialkonstruktivistischen Theorien (Reusser, 2006; Reusser \& Pauli, 2010) betrieben. Das heisst auf der einen Seite, dass davon ausgegangen wird, dass Unterricht eine eigene soziale Wirklichkeit generiert, und auf der anderen, dass der Gegenstand «Sprache» in der sozialen Interaktion mit einer Expertin oder einem Experten wie auch anhand von Aufgaben erworben wird. Expertenstatus können dabei sowohl die Lehrperson als auch kompetentere Mitschülerinnen oder Mitschüler haben. Unter diesen Vorzeichen wird dem Lehrhandeln und dem ihm vorausgehenden didaktischen Plan (Edmondson, 2006) eine besondere Bedeutung beigemessen. Damit einher gehen eine fragend-offene Haltung in Bezug auf die Gestaltung von Unterricht auf der Ebene von Sozialformen, also der Lektionsoberfläche, (Lipowsky, 2006) und in Bezug auf tiefenstrukturelle Merkmale der Spracharbeit innerhalb von Sozialformen wie dem Klassenunterricht (Schwerdtfeger, 2003). 
Für die Umsetzung der Studie war das Angebot-Nutzungs-Modell der Unterrichtqualität und -wirksamkeit ein hilfreiches Konstrukt. Über dieses Modell können Lehr-Lern-Prozesse in der Qualität und in der Quantität beschrieben und Wirkungen von Unterricht nach fachlichen, überfachlichen und erzieherischen Aspekten sowie Sozialisationseffekten ausdifferenziert werden (Reusser \& Pauli, 2010). Als Referenzrahmen für das Erforschen von Unterrichtsqualität insgesamt hat sich die Arbeit mit dem unten abgebildeten Hamburger 4-Stufenmodell (Pietsch, 2013) als zielführend erwiesen.

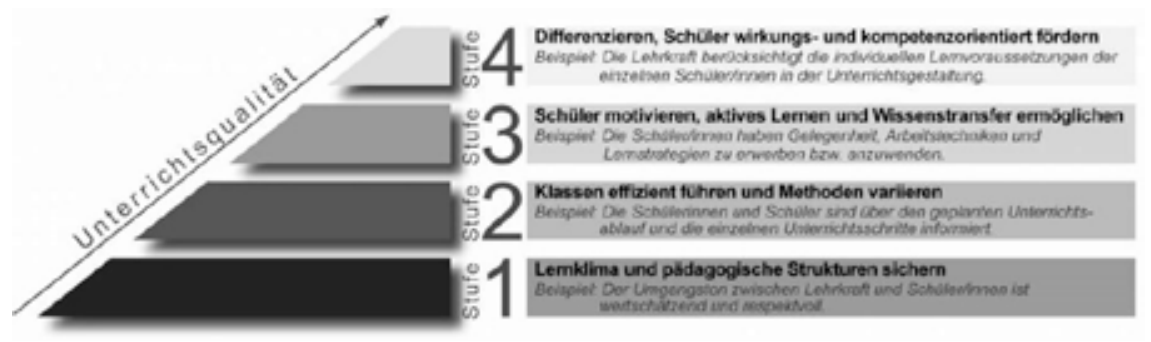

Abbildung 1: Hamburger 4-Stufenmodell zur Unterrichtsqualität (Pietsch, 2013, S. 26).

Dieses Modell erfasst Unterrichtsinteraktionen auf vier Qualitätsstufen: Stufe 1 beschreibt das Lernklima und die pädagogischen Strukturen, Stufe 2 die Klassenführung und die Variation von Methoden, Stufe 3 den aktiven Wissenstransfer und Stufe 4 die kompetenzorientierte Differenzierung. Auf diesen vier Stufen wurden die Untersuchungsmerkmale gemäss dem Didaktischen Dreieck (Reusser \& Pauli, 2010) in Merkmale der Wissens- und Lernkultur und Merkmale der Beziehungs- und Unterstützungskultur unterschieden. Merkmale der Wissens- und Lernkultur sind z.B. Unterrichtsemotionen als Ergebnis eines Lehr-Lern-Prozesses, Merkmale der Beziehungs- und Unterstützungskultur z.B. die Quantität und die Qualität des lehrseitigen Inputs in der Fremdsprache in einer bestimmten Sozialform. Im Forschungsprozess dienten die Modelle zunächst dazu, Untersuchungsmerkmale theoretisch zu verorten. Am Schluss wurden die Forschungsergebnisse dann in die Modelle zurückgeführt, um sie später für die Unterrichtsentwicklung fruchtbar machen zu können.

Ausgewählte empirische Befunde zu den vier Unterrichtsqualitätsstufen zeigen für Stufe 1, dass Emotionen und damit auch das Klima insbesondere für jüngere und schwächere Lernende bedeutsam sind (Meyer, 2004, S. 53) und dass sich bei den Lernenden ein Sozialisationseffekt einstellt, der strukturfördernd sein kann, wenn sie Kommunikationsmuster aus dem Klassenunterricht in Schülerarbeitsformen transferieren (Webb, 2009). Bei der Klassenführung auf Stufe 2 wirkt sich das häufige Hören der Fremdsprache leistungsfördernd aus (DESI-Konsortium, 2006) und zu Gesprächen als Unterrichtsmethode für den fachlichen 
Wissenstransfer auf Stufe 3 gibt es Hinweise, dass sich ein Wechsel zwischen instruktiven und konversationellen Diskursformen für das Durchdringen von inhaltlichen Konzepten als wirksam erweist (Saunders \& Goldenberg, 2007). Während in der Literatur um die Jahrtausendwende hinsichtlich von Stufe 4 ein differenzierender Unterricht noch als die Pädagogik der Zukunft bezeichnet wurde (Gillig, 2001), wird derzeit darauf verwiesen, dass bei der Aneignung von Fachwissen verstärkt auch wieder kollektive Lehr-Lern-Prozesse in den Blick zu nehmen seien (Prediger et al., 2013).

Aus den theoretischen Überlegungen und den empirischen Befunden ergeben sich für die Fallstudien drei Arbeitsfragen:

- Wie bindet die Lehrperson schülerseitiges Vorwissen in das Unterrichtsgespräch ein und um welches Vorwissen handelt es sich dabei?

- Welche fremdsprachlichen Teilkompetenzen werden neu aufgebaut und wie entwickeln sich diese Kompetenzen?

- Wie erleben die Lernenden ihre Kompetenzen und welche unterrichtlichen Zusammenhänge können hierfür geltend gemacht werden?

\section{Untersuchungsdesign}

In der Studie wurden acht Primarschulklassen untersucht, in denen Französisch als zweite Fremdsprache im zweiten Lernjahr unterrichtet wurde. Zunächst wurden die Lehrpersonen in einem Fragebogen zur Situation in ihren Klassen befragt, dann wurden die Klassen in einer Sequenz von drei Lektionen zu Beginn einer unité aus dem Lehrmittel ENVOL 6 videografiert. Nach den Videoaufnahmen wurden die Lernenden in einem Fragebogen zum Unterrichtserleben in den drei Lektionen befragt. Je drei von ihnen, welche die Lehrperson im Unterricht als aktiv erlebt hatte, gaben in einem Gruppeninterview stellvertretend für die Klasse Auskunft zu einzelnen Unterrichtssequenzen, die sie sich am Computer ansehen konnten. Die Auswahl der zu kommentierenden Sequenzen erfolgte durch die Forscherin, welche den Unterricht selbst gefilmt hatte und darin Lehrprozesse beobachtet hatte, die sich kompetenzunterstützend bzw. kompetenzhemmend auf das Lernen auswirkten. Die Befragungs- und Auswertungsinstrumente waren Weiterentwicklungen aus einer schweizerisch-deutschen Videostudie (Klieme et al., 2005), welche für den Fremdsprachenunterricht adaptiert worden waren (Imgrund, 2015).

Die Videodaten wurden auf zwei Ebenen standardisiert: Die gefilmten Lektionen begannen alle mit der Einführung in eine unité, wobei jeweils zwei Lehrpersonen die gleiche unité unterrichteten. Die Auswertungen bezogen sich somit in allen acht Klassen auf den gleichen Lernstand und in je zwei Klassen auf den gleichen Lerngegenstand. Gleichzeitig war davon auszugehen, dass die Kluft zwischen Nichtwissen und aufzubauenden Kompetenzen zu Beginn einer unité relativ hoch war und die Kompetenzentwicklung daher gut beobachtet werden konnte. Die Zusammenstellung der Kontextdaten erfolgte kriteri- 
engeleitet. So nahmen z.B. grosse und kleine Klassen sowie Lehrpersonen mit unterschiedlichem Sprachkompetenzniveau an der Untersuchung teil.

Der Zeitraum der Datenerhebung erstreckte sich von Herbst 2008 bis Frühjahr 2009 (Einzelheiten zu Design und Datensatz in Imgrund, 2015).

Abbildung 2 zeigt die Datenerhebung im Überblick.

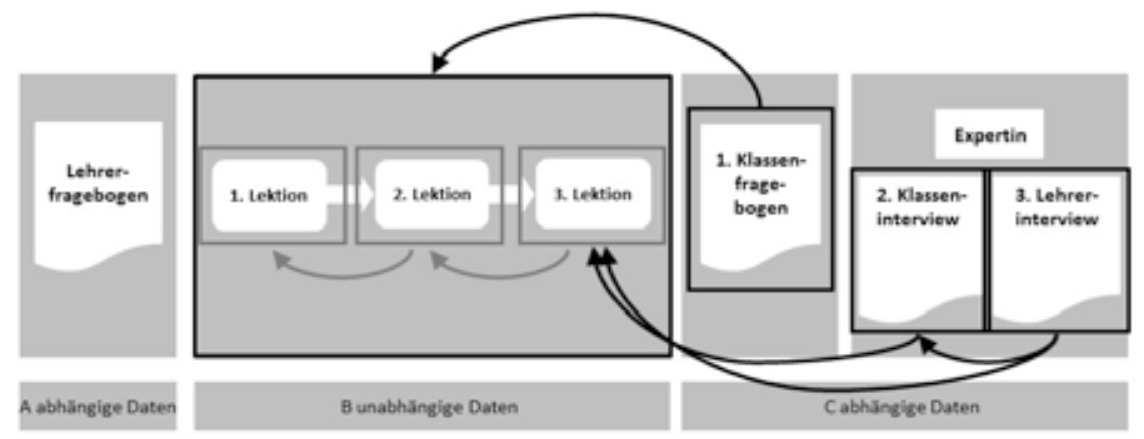

Abbildung 2: Übersicht über die Datenerhebung.

\section{Lehrertypen und Unterrichtsgestaltung}

In einem komplexen Typenbildungsprozess (Imgrund, 2015) konnten für den Primarschulunterricht in Französisch als Fremdsprache verschiedene Lehrertypen identifiziert werden. Theoretisch sind die Typenbezeichnungen im Didaktischen Dreieck verankert und zeigen die Nähe oder die Distanz zum Unterrichtsgegenstand bzw. zu den Lernenden an. Im Folgenden werden exemplarisch der «Steuermann» und der "FremdeSprachenlehrer» vorgestellt. ${ }^{2}$ Hierzu werden zunächst die Unterrichtskontexte skizziert.

\section{Unterrichtskontextmerkmale}

Gemäss dem Lehrerfragebogen scheinen gewisse Voraussetzungen in den Klassen des Steuermanns und des FremdeSprachenlehrers ähnlich zu sein. Die Klassen haben 20 bzw. 21 Lernende, in beiden verläuft der Unterricht nicht immer störungsfrei und es herrscht ein eher schlechtes Klassenklima. Bei der Lehrer-Schüler-Beziehung schildern die Lehrpersonen die Zusammenarbeit mit ihren Klassen als eher mühsam bzw. mühsam, wobei der FremdeSprachenlehrer im Textfeld des Fragebogens noch anfügt, dass seine Klasse geschwätzig sei, einige Querköpfe habe und eine starke Führung benötige. Doch es zeigen sich auch Unterschiede: Während es in der Klasse des FremdeSprachenlehrers Lernende mit anderen Muttersprachen gibt (Albanisch, Bulgarisch, Englisch, Italienisch und Tamil), sind in der Klasse des Steuermanns keine anderen Muttersprachlerinnen und Muttersprachler. Ein Schüler hat jedoch längere Zeit in den USA 
gelebt. Die Klasse des FremdeSprachenlehrers hat eher wenig, die Klasse des Steuermanns grosses Interesse am Fach Französisch. Die Leistungsfähigkeit der Lernenden ist in der Klasse des FremdeSprachenlehrers eher gering, in der Klasse des Steuermannes ausgeprägt. Beide Lehrpersonen haben grosses Interesse an ihrem Fach, verfügen über Fremdsprachenkompetenzen auf B2-Niveau und eine Ausbildung als Primarschullehrperson. Die Mehrsprachigkeitsdidaktik als Sprachlehr- und -lernkonzept der Fremdsprachendidaktik ist beiden ein Begriff.

Pro Lehrertyp werden nachfolgend Transkriptauszüge aus dem Aufbauzyklus in einer Synopse dargestellt, um dann den klassenseitige Nutzen beschreiben und mit Auszügen aus dem Gruppeninterview triangulieren zu können. Danach wird auf die Qualität des Unterrichtsangebots im Aufbauzyklus und schliesslich auf die Kompetenzentwicklung zwischen Aufbau- und Festigungszyklus eingegangen.

\section{Steuermann}

Das folgende Unterrichtstranskript (Beispiel 1) zeigt einen Auszug aus dem Aufbauzyklus, in dem der Steuermann einen Erstkontakt mit dem Lerngegenstand «Französisches Alphabet» herstellt. Die Lernenden bringen Papierbuchstaben, die bereits auf ihren Pulten liegen, nach vorn und heften sie in der richtigen Sequenz an die Tafel. Dann sprechen sie das französische Alphabet im Chor.

\section{Beispiel 1: Instruktiv-konversationeller Wechsel der Diskursform in Passung zum Vorwissen und zu den besonderen Schwierigkeiten des Lerngegenstands 3}

$\mathrm{T}$ Il y a quelques personnes qui ont une lettre sur le pupitre. (..) Vous venez et vous mettez la lettre au tableau noir. [LP macht mit einer Hand eine einladende Bewegung Richtung Wandtafel] (..) [Die Schülerinnen und Schüler, die einen Buchstaben auf dem Pult liegen haben, gehen nach vorn zur Wandtafel und befestigen ihren Buchstaben in der richtigen Reihenfolge. Das Alphabet an der Wandtafel ist am Schluss komplett, bis auf den Buchstaben O, der fehlt] Mais faites attention (..) vous venez. [LP macht mit dem Kopf eine Bewegung in Richtung Wandtafel] Vous venez. MICHĖLE tu prends la lettre de (..) merci. Et vous prenez un stylo. Aujourd'hui nous apprenons l'alphabet (..) en français. Vous n'écrivez rien. (..) Vous écoutez et vous répétez (..) A [LP zeigt immer auf den Buchstaben, den sie gerade ausspricht und den die Schülerinnen und Schüler nachsprechen].
E A
T B
E B
$\mathrm{T} C$
E C
$[\ldots]$ 
$\mathrm{T}$ Nous écrivons quelques aides pour la prononciation. (..) $\mathrm{X}+\mathrm{A}$ c'est comme en allemand c'est A B C D. Mais attention ça c'est E [LP zeigt auf den Buchstaben E] X+ alors vous écrivez ö. [LP schreibt ein kleines ö neben den Buchstaben E]. Vous écrivez ö.

$S$ ? Sie, kommt da bei dem

$\mathrm{T}$ Et on peut dire $\mathrm{E}$ accent aïgu ou bien e accent grave hein. [LP holt zwei Bilder, die bereits auf der Seite an der Wand aufgehängt sind, nach vorn und befestigt sie an der Wandtafel. Auf dem einen Bild sieht man ein é und es steht geschrieben: «l'accent aïgu, l'unité, le thé, le vélo». Auf dem zweiten ist ein è abgebildet mit folgendem Text: «l'accent grave, la mère, la rivière, la règle»]. Nous prenons les images. (..) Voilà. Ou bien seulement $\mathrm{E}$ ça va aussi. Et (..) G (..) hmm. [X+ LP schreibt «sche» neben den Buchstaben G] Et puis le $\mathrm{H}$. [LP schreibt neben den Buchstaben $\mathrm{H}$ «asch»] (..) Et maintenant (..) refléchissez (..) en anglais. [LP klopft mit einem Finger auf den Buchstaben H] Hmm (..) Marco?

S3 H [spricht den Buchstabe englisch aus].

$\mathrm{T}$ Exactement hein? (..) C'est comme un peu en français $\mathrm{H}$ ou bien $\mathrm{H}$ hein en français. I (..) J [X+ LP schreibt neben den Buchstaben J das Wort «schi» hin] (..) T K L M N O P (..) Q [X+ LP schreibt das Wort «qü» neben den Buchstaben Q] R S T U [X+ LP schreibt neben den Buchstaben U den Buchstaben ü hin] et puis $\mathrm{V}$ [X+ LP schreibt das Wort «we» neben den Buchstaben V]. (..) Vous vous souvenez? Lukas?

S10 Double V [Der Schüler spricht das Wort mit englischer Aussprache aus] $Z_{+}$

T Oui double V. [X+ LP schreibt «double-we» neben den Buchstaben W] (..) Nous avons le monsieur Bush hein (..) en Amérique ils appellent? Aussi quelque chose avec le W mais en anglais (..) Petra?

S21 W [Die Schülerin spricht den Buchstaben englisch aus]. [LP nickt]

$\mathrm{T} X(..) \mathrm{Y}[\mathrm{X}+\mathrm{LP}$ schreibt «igrek» neben den Buchstaben $\mathrm{Y}]$ et $\mathrm{Z}$ (..) voilà. [X+ LP schreibt «zet» neben den Buchstaben Z].

Für das Französischlernen besteht der beobachtete schülerseitige Nutzen aus diesem Lernzyklus zunächst darin, dass die Lernenden am Lehr-Lern-Prozess sprachhandelnd aktiv mitwirken. Indem sie die Buchstaben des Alphabets an die Tafel hängen, legen sie die Basis für das nachfolgende Lernen im Klassenverband. Am Ende des Zyklus haben alle die Buchstaben einmal auf Französisch durchgesprochen, ihre Mitlernenden dabei gehört und die phonetischen Besonderheiten des französischen Alphabets kennengelernt.

Beispiel 2 aus dem Gruppeninterview belegt, dass die Lernenden dieses Arrangement der Kompetenzunterstützung als motivierend erlebten. ${ }^{4}$ 


\section{Beispiel 2: Inhaltlicher Beitrag zum Lehr-Lern-Prozess im Aufbauzyklus durch Wahl der Lernunterstützung}

I Kann man das mal mit Beispielen belegen, jetzt aus dieser letzten, aus dieser letzten Lektion? Was ist denn gut? [...]

S1 Also, ja. Und auch, dass wir selbst mal etwas machen können, dass nicht schon alles steht. Dass wir auch selbst, ja

I An was denkst du konkret?

S1 Ja eben, auch das mit dem, mit dem Buchstaben nach vorne bringen.

Mit Blick auf das lehrseitige Angebot auf den vier Unterrichtsqualitätsstufen können im Aufbauzyklus folgende Zusammenhänge identifiziert werden: Der Steuermann stellt die pädagogischen Strukturen über die sequenzielle Struktur des Lerngegenstands $A B C$ und eine angemessene Methode her, indem er die Lernenden altersangemessen physisch aktiviert (Stufe 1). Er hat eine dynamisch klare Klassenführung und erteilt einen variantenreichen Klassenunterricht, der - beim Aufkleben der Buchstaben an die Wandtafel - aufgabengesteuert und beim Erlernen der französischen Aussprache - lehrergesteuert ist (Stufe 2). Den fachlichen Wissenstransfer unterstützt er durch die angemessene Einbindung von kollektiven schülerseitigen Vorkenntnissen sowie durch den Aufbau von neuen, sprachspezifischen Teilkompetenzen wie der Aussprache. Er markiert grafische Besonderheiten des französischen Alphabets (é und è) durch Visualisierungen und gibt den Lernenden für ausgewählte Buchstaben, die im Französischen anders ausgesprochen werden, unter Rückgriff auf ihre Vorkenntnisse aus dem Deutschen und Englischen grafische Aussprachehilfen bzw. fordert die Klasse auf, die Buchstaben auf Französisch nachzusprechen (Stufe 3). Bei den Buchstaben, die nach vorn zu bringen sind, kann er insbesondere die schwächeren Lernenden berücksichtigen, während er bei der Einführung einzelner Buchstaben die kollektiven Vorkenntnisse aus dem Englischunterricht aufnimmt und die besonderen Vorkenntnisse desjenigen Schülers, der in den USA gelebt hatte, differenziert in den Lehr-Lern-Prozess einbaut. Das heisst, dass er in seinem Unterricht entlang der Herausforderungen des Unterrichtsgegenstands kollektive und individuelle Vorkenntnisse der Lernenden in den Lehr-Lern-Prozess einbezieht und seiner Klasse auch im Klassenunterricht ein sehr differenzierendes Lernangebot unterbreitet (Stufe 4).

Im Festigungszyklus steigert die Lehrperson die kognitiven Anforderungen. Sie zeigt einem ersten Schüler ein Wort aus der unité, während ein zweiter Schüler hinter der Tafel steht. Schüler 1 muss das Wort buchstabieren und diktiert es zugleich Schüler 2 und der Klasse. Schüler 2 muss die Buchstaben des Wortes verstehen und an die Wandtafel schreiben, derweil auch die Klasse versucht, die diktierten Buchstaben im Wortkontext zu verstehen und das Wort hörend und schreibend zu erraten. Das Ergebnis des Schülers an der Wandtafel wird danach 
mit dem Ergebnis der Klasse abgeglichen. Im Gruppeninterview (Beispiel 3) berichten die Lernenden von einem kognitiv aktivierenden Lernangebot. Gerade das Diktieren und die kognitiv herausfordernde und verantwortungsvolle Arbeit an der Wandtafel erlebten sie als anregend.

Beispiel 3: Wabl der Lernunterstützung mit anspruchsvoller und verantwortungsvoller Aufgabe an der Wandtafel im Festigungszyklus

\section{S2 Ja und mit dem Diktieren und so}

I Welches Diktieren und so?

S2 Als sie die Buchstaben, also als jemand nach vorne ist und dann die Buchstaben diktiert hat. Und dann mussten wir aufschreiben, was für ein Wort es gibt

S1 ja, das war auch gut

[...]

I Habt ihr noch andere Situationen im Kopf, die euch speziell Spass gemacht haben in den letzten drei Lektionen?

S1 Das Diktieren also, in dieser Lektion, in der letzten. Das Diktieren, dass ein paar nach vorne konnten, jemand an die Wandtafel und dann aufschreiben.

I Was willst du denn sagen?

Ss Die Lösung.

I Aber es reicht doch, wenn du sie für dich hast.

S1 Ja, nein dann wenn's jemand nicht weiss, dann weiss er's nachher S2 von dir persönlich.

I Aha und du hast noch gesagt, der Frau 08 willst du's auch noch sagen?

S1 Ja

I Warum?

S1 Weiss nicht

S3 Ja, sie weiss, dass

S1 dass wir's richtig haben. Dass wir's können.

Mit Blick auf die Kompetenzunterstützung verweist Beispiel 3 auf weitere interessante Zusammenhänge, nämlich darauf, wie Lernende einer 6. Primarklasse ihre Kompetenzen durch die Unterrichtsgestaltung im sozialen Prozess selbst erleben. Sie tun dies, indem sie eine Aufgabe bearbeiten, in der Klassengemeinschaft einen Beitrag zum Lösen der Aufgabe leisten und der Lehrperson den Nachweis erbringen, dass sie es «richtig» können.

Zwischen den zwei Lernzyklen steigt der Grad der kognitiven Aktivierung durch die quantitative linguistische Steigerung vom Buchstaben zum Wort und durch die spracherwerbsrelevante Qualitätserhöhung vom Nachsprechen zum Hörverstehen mit inkludierter Schreibproduktion in einem grösseren fremd- 
sprachigen Sprachhandlungsraum. In diesem Sprachhandlungsraum steuert die Lehrperson den Lehr-Lern-Prozess weiterhin, indem sie bestimmte, im Fremdsprachenunterricht gelernte Wörter zum Buchstabieren vorgibt, welche sie individuell angepasst - in der Schwierigkeit nochmals differenzieren kann.

\section{FremdeSprachenlehrer}

Auch der FremdeSprachenlehrer führt in seinem Unterricht das Alphabet ein. Zu Beginn des Lernzyklus arbeitet er besonders mit denjenigen Lernenden, die eine andere Muttersprache haben, und lässt anschliessend das Alphabet im Chor auf Deutsch aufsagen, bevor die Klasse das Gleiche auf Französisch tut (Beispiel 4).

\section{Beispiel 4: Instruktives Lehren in einer Klasse mit Problemen im Lernklima}

$\mathrm{T}$ Z- Aujourd'hui (..) nous apprenons l'alphabet en français (..). Tu sais l'alphabet en bulgare? [LP zeigt mit der Hand auf einen Schüler]

$S$ ? Je ne sais pas.

$\mathrm{T} \mathrm{Z}$ - Tu ne sais pas et en tamoul? Tu sais l'alphabet en tamoul? [LP zeigt mit der Hand auf Schülerin]

S? Martina.

S18 Ja komm schon. [Schülerin (S18) zählt das gesamte tamilische Alphabet Buchstabe für Buchstabe auf. Ein Schüler klatscht]

$\mathrm{T}$ Ce sont combien lettres?

S18 Ähm treize.

$\mathrm{T}$ Treize (..) et en italien ? [LP zeigt auf einen Schüler]

S3 Z- Je ne sais pas.

$\mathrm{T}$ Tu ne sais pas?

S3 Ja

T A B C.

S3 Äh weisch nid.

$\mathrm{T}$ Et en anglais? (..) MARTIN tu sais l'alphabet en anglais?

S19 Z- Non

$\mathrm{T}$ Essaie.

$\mathrm{T}$ Z+ A (..) A [Die LP spricht den Buchstaben zuerst auf Französisch und dann auf Englisch aus]

S19 A B C D E F G I [Schüler spricht das I falsch aus]

$\mathrm{T}$ I

S19 I J K

$[\ldots]$

T X Y Z

S19 X Y Z

T X Y Z bien. En Colom = ah en albanais tu sais l'alphabet en albanais? 
S? Z- Je ne sais pas.

$\mathrm{T}$ Rien?

S? Ich auch nicht.

T Pas? (..) Aussi pas? Tu sais alors en allemand? [LP schaut zu einer Schülerin]

S? Ja ich kann's nicht.

$\mathrm{T}$ Alors toute la classe en allemand hein (..) toute la classe. [LP macht mit beiden Armen eine kleine Halbkreisbewegung von unten nach oben, um die gesamte Klasse aufzufordern]

E X-A B C D E F G H I J K L M N O P Q R S T U V W X Y Z. [Die Klasse zählt das deutsche Alphabet auf und krakeelt dabei. Ein Schüler stört die gesamte Klasse, indem er die Buchstaben so ausspricht, wie man sie schreibt. Er spricht immer, nachdem die Klasse den Buchstaben ausgesprochen hat]

$T$ Voilà. [LP geht zur Wandtafel und klappt eine neue Tafel auf. Folgendes Tafelbild kommt zum Vorschein: «A, B, C, D, E, F, G, H, I, J, K, L, M, N, O, P, Q, R, S, T, U, V, W, X, Y, Z»] [Schülerinnen und Schüler wiederholen die Buchstaben auf Deutsch, lachen laut und unterhalten sich] Maintenant écoutez en français hein? (..) A [LP zeigt mit dem Zeigestab auf den Buchstaben A an der Wandtafel]

Ss A

E A

T B [LP zeigt mit dem Zeigestab auf den Buchstaben B]

E B

$[\ldots]$

$\mathrm{Zu}$ Beginn dieses Lernzyklus sind eine reduzierte Lernzeit und damit ein geringer Nutzen für die ganze Klasse beobachtbar, weil sich die Lehrperson zunächst sehr eingehend mit einzelnen Lernenden beschäftigt. Danach sagt die Klasse das Alphabet erst auf Deutsch auf und lernt anschliessend die französische Aussprache der Buchstaben. Der aktive Einbezug des schülerseitigen Vorwissens aus anderen Sprachen gelingt nicht. Die Klasse zieht allerdings einen ganz eigenen «Nutzen» aus dem Unterricht, indem sie die Kooperation mit der Lehrperson geschickt verweigert und lernhemmende Kommunikationsmuster von Mitlernenden nachahmt.

Mit Blick auf das Unterrichtsangebot des FremdeSprachenlehrers können auf den vier Unterrichtsqualitätsstufen folgende Zusammenhänge ausgemacht werden: Das bereits schlechte Klima in der Klasse verstärkt er durch eine gleichförmige Klassenführung im Unterrichtsgespräch. Durch die nicht gelingende Zusammenarbeit mit einzelnen Lernenden beeinträchtigt er die pädagogischen Strukturen (Stufe 1). Auch sein Klassenunterricht ist gleichförmig und vorwiegend lehrergesteuert (Stufe 2). Durch eine kognitiv zu tief angesetzte Unterstützung bei der Einbindung von kollektiven Wissensbeständen (Buchstabieren des deutschen Alphabets im Chor) hemmt er die Kompetenzentwicklung 
und erschwert einen differenzierten Aufbau von Teilkompetenzen im Französischen (Stufe 3). Zudem wendet er eine lernhemmende Differenzierung an, indem er einzelne Lernende und ihre Herkunftssprachen zu Beginn des Lehr-Lern-Prozesses exponiert und dabei den Fokus auf die Zielsprache Französisch verliert (Stufe 4).

Der folgende Auszug aus dem Gruppeninterview (Beispiel 5) zeigt, wie stark die Unterrichtsqualität auf den Stufen 1 und 2 mit dem fachlichen Lehr-LernProzess zusammenhängt. Das oben dargestellte Unterrichtsereignis kommentieren die Lernenden wie folgt.

\section{Beispiel 5: Einschränkungen beim Herstellen von pädagogischen Strukturen durch die Klassenführung}

S3 Französisch spricht er gut, wie ich auch im Fragebogen ausgefüllt habe. Aber nur ein Problem ist, das, was er ausspricht, die Hälfte versteht niemand und dann fragt man nach, wa- was es heisst, und dann kann man auch wieder dazulernen und was er nicht gut macht, ist eben immer nur auf eine Person. Wenn diese Person spricht, schaut er nur auf diese, und dann können die anderen die Sau rauslassen [...] dann ist er nur noch auf dieser Person und manchmal finde ich, er hat nur Kontrolle über eine Person, nicht über die ganze Klasse.

Mit Blick auf den aktiven Wissenstransfer auf Unterrichtsqualitätsstufe 3 ist festzustellen, dass die Lernenden auch den Lerngegenstand $A B C$ nicht als kognitive Herausforderung ansahen. Die folgenden Auszüge aus dem Gruppeninterview (Beispiele 6 und 7) geben Auskunft darüber, wie die Lernenden die Gestaltung des Unterrichts in einem mangelhaften Lernklima und mit einem reduzierten kognitiven Anspruchsniveau erleben und welche «Kompetenzen» zur Kompensation sie im Unterrichtsgespräch entwickeln (Beispiel 8).

\section{Beispiel 6: Angemessenheit des kognitiven Anspruchsniveaus}

I Für dich, wie ist es, wenn du eine andere Sprache hörst?

S3 Auch etwas Neues, aber ich finde es es bringt nichts. Ich meine ich kann nicht, wenn ich das ABC auf Tamilisch höre, kann ich nicht mit M. tamilisch kommunizieren, oder. Man sollte schon ganze Sätze sagen können. Und dann auf Deutsch übersetzen wie zum Beispiel «Hallo ich heisse M.» [...]

\section{Beispiel 7: Geringe kognitive Herausforderung beim Buchstabieren und hemmendes Lernklima in der Lerngruppe \\ I Also, das ist so da eine, dass man die anderen hört, [...] und für dich persönlich, als du das englische Alphabet gesagt hast, als du aufgerufen wurdest, wie war das und für dich persönlich, als du aufgerufen wurdest? [...]}


S3 Wo er nach dem englischen Alphabet gefragt hat, war es auch so irgendwie «Nein jetzt muss ich das auf Englisch sagen», oder so und es kann jeder, es ist fast mit dem mit der deutschen Sprache verwandt oder wie A B C. Nur es gibt ein paar Buchstaben, [...] die sind ein bisschen anders beim Aussprechen und so. [...] Ja für mich war es sehr eklig, weil alle zuhörten und alle schauten mich an und so.

Beispiel 8: Partizipation als lehrseitige Aufforderung zur Mitarbeit und schülerseitige Kompensationsstrategien zur Verweigerung

I [...] das hab ich auch beobachtet. Manchmal nimmt er ja jemanden dran und sagt: «Du wiederholst das jetzt nochmals.» Wie ist das für euch, wenn man dann so «So, jetzt du wiederholst das nochmals». [...]

S3 Wenn ich in dieser Situation wäre, wäre es für mich ein bisschen heikel, weil weil alle wissen ja, der hat nicht zugehört und und er weiss jetzt nicht, was sagen, dann ist man so still, oder. Dann sagt man irgendwie «Nein, ich weiss nicht die Lösung», und dann muss der Lehrer sie wieder sagen $[\ldots]$

Das schlechte Lernklima unter den Lernenden und die schwierigen pädagogischen Strukturen äussern sich in dieser Klasse im Festigungszyklus noch drastischer. Bei einem grösser werdenden Freiheitsgrad im Sprachhandlungsraum dürfen die Lernenden der Lehrperson ein selbst gewähltes Wort zurufen. Die Lehrperson schreibt dieses an die Tafel und ruft dann einen anderen Schüler oder eine andere Schülerin auf, der bzw. die es buchstabieren soll. Aus der Beobachterperspektive arbeiteten die Lernenden dabei sehr eifrig mit. Wie der Auszug aus dem Gruppeninterview (Beispiel 9) jedoch zeigt, ist die Schülerperspektive auf das gleiche Unterrichtsereignis eine andere.

\section{Beispiel 9: Schlechtes Klima unter den Schülerinnen und Schülern}

I Dann hat der Herr 07 ja Sachen von euch an die Tafel geschrieben, erinnert ihr euch? Wie war das für euch? Wörter von euch, die ihr diktiert habt, wie war das für euch? Als er die Sachen an die Tafel geschrieben hat?

S3 Man kann auch so ein bisschen die Klasse ärgern mit schwierigen Wörter und dann ruft er einfach eine Person auf und dann ist er das Opfer, weil er es buchstabieren muss, dann hat man ein bisschen Schadenfreude, wenn er etwas falsch sagt oder so und manchmal kommt es einfach freiwillig, man streckt automatisch und sagt irgendwie ein Wort.

Mitlernenden wird demnach mitunter bewusst ein schwieriges französisches Wort vorgegeben, welches sie dann vor der ganzen Klasse öffentlich buchstabieren müssen. Der Unterrichtsgegenstand «Fremdsprache» wird nicht seriös behandelt, sondern willentlich zur Störung des Unterrichts bzw. für eigene Zwecke genutzt. Beobachtbar war dies in dieser Klasse ebenfalls, wenn die Frage 
nach dem Befinden sehr keck mit «Ça va mal» beantwortet wurde oder einer nach dem anderen sehr höflich fragte: «Puis-je aller au WC?»

Mit Blick auf die Kompetenzunterstützung können die Lernenden zwischen den Zyklen in einem grösseren Sprachhandlungsraum zwar eine Entwicklung des Gegenstands erleben, dieser birgt im Aufbauzyklus jedoch noch fachliche Unschärfen bei den fremdsprachenspezifischen Besonderheiten des Französischen und hat aus Klassensicht wenig Relevanz. In beiden Zyklen wird der Gegenstand zudem volitional eher widerwillig erarbeitet und sozial z.T. missbraucht.

\section{Qualitätsvolles Lehrhandeln im elementaren Französischunterricht}

Wie die beiden Fallstudien zeigen, kann die Kompetenzunterstützung der Lehrperson als wichtiger Parameter für die Entwicklung von fachlichen und überfachlichen Kompetenzen angesehen werden. Dabei zeichnet sich die Arbeit im Klassenzimmer insbesondere durch zwei Dimensionen der Kompetenzunterstützung aus, nämlich die methodisch passgenaue Integration von Vorwissen und den angemessenen Aufbau von neuem Wissen. Während die Lernenden des Steuermanns durch passende inhaltliche Beiträge am Lehr-Lehr-Prozess partizipieren und neue Kompetenzen aufbauen können, gelingt dies im Unterricht des FremdeSprachenlehrers beim gleichen Lerngegenstand wenig.

Mit Blick auf das schülerseitige Vorwissen und seine Einbindung in den Lehr-Lern-Prozess kann zwischen vorhandenen Sprachkompetenzen in der Herkunftssprache, kollektivem Vorwissen aus dem Englisch- und Deutschunterricht sowie individuellem Vorwissen einzelner Lernender unterschieden werden. Dieses Vorwissen wird vom Steuermann je nach Lernstand der Klasse und in Passung zum Lerngegenstand methodisch kreativ aktiviert. Dabei behält er stets den kollektiven Lernprozess im Blick und treibt ihn fachlich präzise voran. Der FremdeSprachenlehrer hingegen wendet das Konzept der Differenzierung im Rahmen der Mehrsprachigkeitsdidaktik formelhaft an und verliert dabei das Ziel für die Klasse, nämlich Französisch zu lernen, aus den Augen. Seine gleichförmige Gesprächsführung scheint dabei den klassenseitigen Lernprozess zusätzlich zu hemmen, denn indem die Lernenden ihre Mitschülerinnen und Mitschüler im Gespräch imitieren, stören sie wiederum die pädagogischen Strukturen.

Zum Aufbau von Kompetenzen in einem Zyklus und zur Kompetenzentwicklung zwischen zwei Zyklen konnte festgestellt werden, dass der Fokus der Kompetenzunterstützung im Aufbauzyklus auf dem Erlernen von in quantitativer und qualitativer Hinsicht kognitiv eher wenig anspruchsvollen Teilkompetenzen (z.B. der französischen Aussprache), aber auch auf dem Vermitteln von Sprachspezifika zu liegen scheint. Im Festigungszyklus verschiebt sich der Fokus auf die kommunikative Anwendung dieser Teilkompetenzen. In einem grösser werdenden Sprachhandlungsraum können Fremdsprachenkenntnisse im sozialen 
Umfeld des Klassenzimmers angewendet werden. Sprachdidaktisch gelingt dies beiden Lehrertypen, die Bildungswirkungen sind indes unterschiedlich. Beim Steuermann wird durch die Arbeit am Lerngegenstand ein Lehr-Lern-Prozess in Gang gesetzt, in dem fachliche, volitionale und soziale Kompetenzen gefördert werden. Beim FremdeSprachenlehrer hingegen wird der Lerngegenstand von der Klasse zwar bearbeitet, durch die methodischen Mängel im Unterrichtsangebot werden soziale Defizite und die schwierigen Strukturen in der Klasse aber noch verstärkt.

Beide Lehrpersonen arbeiten in der gleichen Sozialform «Klassenunterricht». In beiden Klassen hören die Lernenden auf der Lektionsoberfläche viel Französisch von der Lehrperson. Ein tiefenstruktureller Vergleich fördert jedoch bedeutsame Unterschiede bei der Gestaltung der schülerseitigen Partizipation, den kognitiven Anforderungen und der Gestaltung des Unterrichtsgesprächs zutage: Beim Steuermann ist die Gesprächsform pro Lernzyklus und innerhalb eines Lernzyklus massgeschneidert. Die instruktive bzw. konversationelle Gesprächsführung ergibt sich aus einer genauen Passung zwischen den Anforderungen des Lerngegenstands und dem Vorwissen der Lerngruppe. Beim FremdeSprachenlehrer hingegen ist sie inhaltlich wenig präzis und methodisch gleichförmig. So kommt es in der Klasse des Steuermannes zu einer positiven und in der Klasse des FremdeSprachenlehrers zu einer negativen Lehr-Lern-Dynamik, woraus jeweils unterschiedliche Fachkenntnisse und - gemäss dem Klassenfragebogen - auch eine unterschiedliche Interessensentwicklung hinsichtlich der Fremdsprache Französisch resultieren. Vereinfacht und mit den Worten der Lernenden ausgedrückt möchte es die Klasse des Steuermanns «richtig haben ... [und] können» (Beispiel 3), während die Klasse des Fremdesprachenlehrers «die Sau rauslassen» will (Beispiel 5).

Was die Gestaltung von Lehr-Lern-Prozessen im Volksschulunterricht der Primarstufe anbelangt, erstaunte die Kleinschrittigkeit bei der Kompetenzunterstützung im Klassenverband. In der Tiefenstruktur betrifft dies die quantitative Progression in der linguistischen Menge (vom Buchstaben zum Wort) und die spracherwerbsrelevante Progression in der Qualität des Outputs (vom Nachsprechen zum Selbersprechen) sowie die Freiheitsgrade im Sprachhandlungsraum der Fremdsprache zwischen zwei Lernzyklen. Während der Steuermann den Lehr-Lern-Prozess lerngegenstands- und lernerbezogen von der Fremd- zur Selbststeuerung sehr differenzierend aktiv lenkt, läuft der Prozess beim FremdeSprachenlehrer, der gerade sehr lernerbezogen arbeitet, aus dem Ruder.

Jenseits von real zu bewältigenden Aufgaben zählen für die schülerseitige Motivation im Klassenzimmer also noch andere Parameter für den Zugang zum Fach. Dies sind soziale Eingebundenheit und Partizipationsmöglichkeiten am Lehr-Lern-Prozess in Verbindung mit einer angemessenen kognitiven Herausforderung und einer präzisen Arbeit am Lerngegenstand. 


\section{Diskussion und Ausblick}

Angesichts der in der Analyse festgestellten formelhaften Anwendung des Konzepts der Mehrsprachigkeitsdidaktik und der daraus folgenden Konsequenzen für die Unterrichtsqualität, die sich im Falle des FremdeSprachenlehrers klar abgezeichnet haben, verdeutlichen die Befunde der Fallstudien, wie wichtig eine sorgfältige Planung in den Tiefenstrukturen von Unterricht ist. Dazu gehört, dass Lehrpersonen die Bedeutung der Aufgaben, ihre fremdsprachenspezifischen Besonderheiten, die Unterschiede und Parallelen zu anderen Sprachen sowie Zusammenhänge mit dem Lerngegenstand analysieren und diesen in den anvisierten fremdsprachlichen Kommunikationskompetenzen verorten. Die Ergebnisse dieser Aufgabenanalyse können sodann auf die Diagnose der besonderen Bedingungen einer Klasse projiziert werden, woraus schliesslich eine passgenaue Methode für die jeweilige Klasse abgeleitet werden kann. Für die Ausbildung von Lehrpersonen dürfte es demnach von Vorteil sein, von der Idee des «Ingenieurs» auszugehen, welcher komplexe inhaltliche und pädagogische Voraussetzungen erfasst, daraus pädagogische und fachliche Ziele ableitet und in der Lage ist, diese Ziele methodisch kreativ umzusetzen.

Die Erkenntnisse aus den beiden Fallstudien sind zwar noch limitiert, werden aber durch andere Hinweise aus der Gesamtstudie gestützt. Mit dem gewählten Untersuchungsdesign konnte theoretisch abgestützt ausserdem aufgezeigt werden, wie die Qualität von komplexen Lehr-Lern-Prozessen im Fachunterricht erforscht werden kann. Die Frage, inwiefern dieser Zugang auf andere fachdidaktische Studien übertragbar ist und wie die Analyse- und Diagnosefähigkeiten von Fremdsprachenlehrpersonen als wichtige Basis für einen differenzierenden Fachunterricht noch genauer erforscht werden können, wäre künftig anzugehen.

\section{Anmerkungen}

1 Die Publikation wurde von der Aebli-Näf-Stiftung gefördert.

2 Um die Erkenntnisse prägnant formulieren zu können, wurden für die Typen generisch maskuline Bezeichnungen gewählt. Ausserdem sollte bereits die Wortoberfläche Aussagen über die Qualität des Unterrichts erlauben. Beim «FremdeSprachenlehrer» handelt es sich um eine Eigenkreation, welche den Typus von der ordentlichen Fremdsprachenlehrperson abheben soll.

3 Erläuterungen zu den Transkriptauszügen: «T» = «Lehrperson» als Turnbezeichnung; « $\mathrm{S}$ » $=$ «Schülerin/Schüler»; «LP» = «Lehrperson» in der Beschreibung; «E» = «Entire class» für die ganze Klasse; «Ss» = «mehrere Lernende aus der Klasse»; «(..)» kennzeichnet Pausen von weniger als drei Sekunden; «X-» = Die Äusserung einer Sprecherin oder eines Sprechers enthält Unregelmässigkeiten im inneren Diskurs, welche für die Lehr-Lern-Kommunikation störend sein können; "X+» = Die Äusserung einer Sprecherin oder eines Sprechers enthält lernunterstützende Hilfen im inneren Diskurs; "Z-» = Das äussere Diskursverhalten ist fachgebunden, schränkt aber die französischsprachige Sprachproduktion der Schülerinnen und Schüler ein; « $\mathrm{Z}_{+} »=$ Eine Sprecherin oder ein Sprecher äussert sich und behält dabei die Diskursstruktur bei.

4 Die Aussagen, auf die in der Interpretation Bezug genommen wird, werden jeweils durch Fettdruck hervorgehoben. 


\section{Literatur}

DESI-Konsortium. (2006). Unterricht und Kompetenzerwerb in Deutsch und Englisch. Zentrale Befunde der Studie Deutsch-Englisch-Schülerleistungen-International (DESI). Frankfurt a.M.: DIPF.

Edmondson, W. (2006). Konversationsanalyse und Lehrerverhalten im Fremdsprachenunterricht. In U. H. Jung (Hrsg.), Praktische Handreichung für Fremdsprachenlehrer (4., vollst. neu bearb. Aufl., S. 51-57). Frankfurt a.M.: Lang.

Europarat. (2001). Gemeinsamer europäischer Referenzrahmen für Sprachen: lernen, lehren, beurteilen. Berlin: Langenscheidt.

Gillig, J.-M. (2001). Les pédagogies différenciées. Origine, actualité, perspective. Brüssel: De Boeck.

Imgrund, B. (2007). Mehrsprachigkeitsdidaktik und ihre Anwendung im sprachlichen Anfangsunterricht. Babylonia, 16, (3), 49-62.

Imgrund, B. (2015). Lehrertypen und Unterrichtsqualität im Fremdsprachenunterricht. Multiperspektivische Fallstudien zur Kompetenzentwicklung und zum Kompetenzerleben im Französischunterricht. Unveröffentlichte Dissertation, Universität Genf.

Klieme, E., Pauli, C. \& Reusser, K. (2005). Dokumentation der Erhebungs- und Auswertungsinstrumente zur schweizerisch-deutschen Videostudie "Unterrichtsqualität, Lernverhalten und mathematisches Verständnis»: Befragungsinstrumente (Band 1). Frankfurt a.M.: DIPF.

Lipowsky, F. (2006). Auf den Lehrer kommt es an. Empirische Evidenzen für Zusammenhänge zwischen Lehrerkompetenzen, Lehrerhandeln und dem Lernen der Schüler. Zeitschrift für Pädagogik, 51, Beiheft, 47-70.

Meissner, F.-J. \& Reinfried, M. (1998). Mehrsprachigkeitsdidaktik. Konzepte, Analysen, Lehrerfahrungen mit romanischen Fremdsprachen. Tübingen: Narr.

Meyer, H. (2004). Was ist guter Unterricht? Berlin: Cornelsen.

Pietsch, M. (2013). Was guten Unterricht kennzeichnet. Bildung \& Wissenschaft, 12, 24-28.

Prediger, S., Komorek, M., Fischer, A., Hinz, R., Hußmann, S., Moschner, B., Ralle, B. \& Thiele, J. (2013). Der lange Weg zum Unterrichtsdesign. Zur Begründung und Umsetzung fachdidaktischer Forschungs- und Entwicklungsprogramme. In M. Komorek \& S. Prediger (Hrsg.), Der lange Weg zum Unterrichtsdesign (S. 9-23). Münster: Waxmann.

Reusser, K. (2006). Konstruktivismus - vom epistemologischen Leitbegriff zur Erneuerung der didaktischen Kultur. In M. Baer, M. Fuchs, P. Füglister, K. Reusser \& H. Wyss (Hrsg.), Didaktik auf psychologischer Grundlage. Von Hans Aeblis kognitionspsychologischer Didaktik zur modernen Lehr- und Lernforschung (S. 151-168). Bern: hep.

Reusser, K. \& Pauli, C. (2010). Einleitung und Überblick. In K. Reusser, C. Pauli \& M. Waldis (Hrsg.), Unterrichtsgestaltung und Unterrichtsqualität. Ergebnisse einer internationalen und schweizerischen Videostudie zum Mathematikunterricht (S. 9-32). Münster: Waxmann.

Saunders, W. M. \& Goldenberg, C. (2007). The effects of an instructional conversation on English language learners' concept of friendship and story comprehension. In R. Horowitz (Éd.), Talking Texts (pp. 221-252). Mahwah, NJ: Erlbaum.

Schneuwly, B. (2009). Die Fachdidaktiken - im Zentrum der Unterrichtsforschung und -entwicklung. Beiträge zur Lehrerinnen- und Lehrerbildung, 27, (3), 313-324.

Schwerdtfeger, I. C. (2003). Sozialformen: Überblick. In K.-R. Bausch, H. Christ \& H.-J. Krumm (Hrsg.), Handbuch Fremdsprachenunterricht (4., vollst. überarb. Aufl., S. 247-251). Tübingen: Francke.

Webb, N. M. (2009). The teacher's role in promoting collaborative dialogue in the classroom. British Journal of Educational Psychology, 79, (1), 1-28.

Weinert, F. E. (2001). Vergleichende Leistungsmessung in Schulen - eine umstrittene Selbstverständlichkeit. In F. E. Weinert (Hrsg.), Leistungsmessungen in Schulen (S. 17-31). Weinheim: Beltz. 
Schlagworte: Fremdsprachenunterricht, Kompetenzunterstützung, Primarstufe, Lehrhandeln, Kompetenzentwicklung

\section{L'enseignement du français langue étrangère à l'école primaire.}

\section{Développement de compétences dans un contexte multiculturel}

Ré sumé

Quelles relations peut-on observer entre la pratique des enseignants et le développement de compétences dans un cours de français langue étrangère? Afin de répondre à cette question, le présent article propose d'abord une sélection de modèles théoriques ainsi quelques résultats empiriques de recherches menées à propos de la qualité de l'enseignement les langues étrangères à l'école primaire. La présentation de l'échantillon de l'étude sur laquelle se fonde la recherche précède ensuite l'analyse descriptive et interprétative de deux exemples dans lesquels des élèves issus de plusieurs cultures et langues (qui ne sont ni l'allemand, ni le français) travaillent dans deux classes différentes sur les même thèmes et avec les mêmes opportunités d'apprentissage, sans en retirer cependant les mêmes bénéfices. Enfin, la comparaison de ces deux cas et les éléments empiriques qui en sont issus permettent d'émettre quelques propositions pour l'enseignement et le développement de compétences dans l'enseignement du français langue étrangère à l'école primaire.

Mots-clés: Enseignement d'une langue étrangère, soutien au développement des compétences, école primaire, pratiques d'enseignement, développement des compétences

\section{Un'istruzione di alta qualità nell'insegnamento del francese La didattica plurilingue e lo sviluppo delle competenze a livello elementare}

\section{Riassunto}

Come si può descrivere in modo più approfondito la qualità dell'agire del docente e lo sviluppo delle competenze nell'insegnamento del francese? Rispondendo a questa domanda si riportano in primo luogo modelli teorici e risultati di ricerche empiriche selezionate sull'istruzione e la glottodidattica. Da un campione di un totale di otto casi vengono poi analizzati due casi in modo descrittivo-interpretativo. Pur affrontando gli stessi contenuti, le due classi ne traggono benefici differenti. Riferendosi a diverse fonti di dati il confronto dei due casi permette di dedurre suggerimenti empiricamente fondati su come sostenere l'agire del docente in un approccio orientato alle competenze nell'insegnamento elementare. 
Parole chiave: Insegnamento delle lingue straniere, supporto allo sviluppo delle competenze, l'agire del docente, scuola primaria

\title{
High-quality instruction in teaching French \\ Multilingual teaching and competence development at primary school level
}

\begin{abstract}
How can we describe high-quality instruction and competence development in the French classroom more profoundly? In order to answer this question, the article first presents theoretical models and a selection of empirical findings from research into instructional quality and foreign language education. Out of an 8-teacher sample the study describes the descriptive-interpretive analysis of two cases in which teachers follow the principles of multilingual teaching. In these cases the pupils work on the same topic in a whole-class setting, but do not derive the same benefit from the learning opportunities offered to them. Based on a comparison between the two cases, the concluding section infers some empirically substantiated suggestions for teaching that fosters competence development in French lessons at the primary school level.
\end{abstract}

Keywords: Foreign language education, competence-oriented learning support, primary school level, teaching practices, competence development 
